\title{
BMJ Open Paramedic attitudes and experiences of enrolling patients into the PARAMEDIC-2 adrenaline trial: a qualitative survey within the London Ambulance Service
}

\author{
Johanna Lazarus, ${ }^{1}$ Rajeshwari lyer, ${ }^{1}$ Rachael T Fothergill (ib) ${ }^{1,2}$
}

To cite: Lazarus J, lyer R, Fothergill RT. Paramedic attitudes and experiences of enrolling patients into the PARAMEDIC-2 adrenaline trial: a qualitative survey within the London Ambulance Service. BMJ Open 2019;9:e025588. doi:10.1136/ bmjopen-2018-025588

- Prepublication history and additional material for this paper are available online. To view these files, please visit the journal online (http://dx.doi. org/10.1136/bmjopen-2018025588).

Received 23 July 2018 Revised 04 October 2019 Accepted 04 November 2019

Check for updates

(C) Author(s) (or their employer(s)) 2019. Re-use permitted under CC BY-NC. No commercial re-use. See rights and permissions. Published by BMJ.

${ }^{1}$ London Ambulance Service NHS Trust, London, UK

${ }^{2}$ Clinical Trials Unit, Warwick University, Coventry, England

Correspondence to Professor Rachael T Fothergill; rachael.fothergill@lond-amb. nhs.uk

\section{ABSTRACT}

Objectives The aim of this study was to gather the views and experiences of paramedics who participated in a large-scale randomised controlled drug trial and to identify barriers to recruitment.

Design We surveyed paramedics using a questionnaire consisting of a mix of closed and open ended questions.

Setting The study was conducted within the London Ambulance Service, London, UK.

Participants 150 paramedics who were trained to enrol patients into the PARAMEDIC-2 randomised controlled trial of adrenaline versus placebo in out-of-hospital cardiac arrest and who returned the questionnaire.

Results $98 \%$ of study participants felt prehospital research was very important, and $97.3 \%$ reported an overall positive experience of being involved in a drug trial. Only $5.3 \%$ felt uncomfortable enrolling patients into the trial without prior consent from the patient or a relative. Over one- third (39.3\%) identified one or more barriers to patient recruitment, the most common being the attitudes of other staff.

Conclusion We found a strong appetite for involvement in prehospital research among paramedics and an understanding of the importance of research that prevailed over the complexities of the trial. This is an important finding demonstrating that potentially ethically controversial research can be undertaken successfully by paramedics in the prehospital environment.

\section{BACKGROUND}

The London Ambulance Service NHS Trust (LAS) was one of five UK ambulance services that participated in the PARAMEDIC-2 trial, a large-scale randomised controlled trial that compared adrenaline to a saline placebo in out-of-hospital cardiac arrest (OHCA). ${ }^{1}$ The trial was undertaken in response to increasing uncertainty about the safety and effectiveness of adrenaline as a treatment for OHCA. ${ }^{2-4}$ Between December 2014 and October 2017, 8014 cardiac arrest patients were enrolled across all sites, making it the largest cardiac arrest drug trial in Europe and the largest
Strengths and limitations of this study

- The study reports on an under-researched area, examining the views and experiences of paramedics conducting a prehospital drug trial.

- The questionnaire was completed anonymously, and therefore, respondents were likely to have been honest and open in their answers.

- As completion of the questionnaire was voluntary, the study could have been influenced by responder bias.

- Our study was limited to paramedics who had successfully completed the PARAMEDIC-2 trial training package and therefore does not represent views of paramedics who chose not to participate in the trial.

- As paramedics were involved in recruiting into the trial that ran for almost 3 years, there may be an element of recall bias that should be considered.

randomised controlled trial of adrenaline in the world. A total of 2102 patients were enrolled by LAS paramedics. This paper focuses on the attitudes and experiences of London paramedics who participated in the trial. Details of the PARAMEDIC-2 trial protocol and the findings from the trial have been reported elsewhere. ${ }^{15}$

As a cardiac arrest renders the patient unable to consent for themselves, and the urgency of the situation makes it unfeasible to seek proxy consent from a relative or friend on scene, ethical approval was granted to the PARAMEDIC-2 trial, enabling paramedics to recruit patients without first seeking consent from either the patient or a representative. Consent for subsequent follow-up was later sought by a member of the research team from patients who survived to be admitted to hospital, or from their personal representative where capacity was not regained. Members of the public who did not want 
to become potential trial patients could 'opt out' by requesting a 'no study' bracelet from the trial Sponsors, and paramedics were trained not to enrol any patient wearing one of these bracelets. ${ }^{5}$

The London Ambulance Service NHS Trust (LAS) was one of five UK ambulance services that participated in the PARAMEDIC-2 trial, a large-scale randomised controlled trial that compared adrenaline to a saline placebo in outof-hospital cardiac arrest (OHCA). ${ }^{1}$ The trial was undertaken in response to increasing uncertainty about the safety and effectiveness of adrenaline as a treatment for OHCA. ${ }^{2-4}$ Between December 2014 and October 2017, 8014 cardiac arrest patients were enrolled across all sites, making it the largest cardiac arrest drug trial in Europe and the largest randomised controlled trial of adrenaline in the world. A total of 2102 patients were enrolled by LAS paramedics. This paper focuses on the attitudes and experiences of London paramedics who participated in the trial. Details of the PARAMEDIC-2 trial protocol and the findings from the trial have been reported elsewhere. ${ }^{15}$ As a cardiac arrest renders the patient unable to consent for themselves, and the urgency of the situation makes it unfeasible to seek proxy consent from a relative or friend on scene, ethical approval was granted to the PARAMEDIC-2 trial, enabling paramedics to recruit patients without first seeking consent from either the patient or a representative. Consent for subsequent follow-up was later sought by a member of the research team from patients who survived to be admitted to hospital, or from their personal representative where capacity was not regained. Members of the public who did not want to become potential trial patients could 'opt out' by requesting a 'no study' bracelet from the trial Sponsors, and paramedics were trained not to enrol any patient wearing one of these bracelets. ${ }^{5}$

Due to the nature of the trial, which involved some patients receiving a saline placebo instead of a drug that has been used for over 50 years to treat cardiac arrest, the trial received media attention, some of which was controversial. ${ }^{6-8}$ Much of the media coverage occurred prior to the start of the study, with controversy generally centred on patients being recruited without their consent and the removal of access to adrenaline for those enrolled into the placebo arm of the trial.

The design of the PARAMEDIC-2 trial required paramedics to independently determine eligibility and randomise patients into the trial by administering the blinded drugs (either adrenaline or a saline placebo) from a trial-specific drug pack. The approach to both participation and trial protocol training varied between ambulance services. Some services made participation voluntary, whereas others mandated that all paramedics participate. Training delivery was achieved either face to face or online, and during working hours or in the paramedic's own time, depending on the individual ambulance service. Within the LAS, we took the approach of voluntary participation, with mandatory training. In other words, paramedics wishing to participate were required to complete a trial training package consisting of a $35 \mathrm{~min}$ DVD presentation (including background evidence, ethical considerations, the trial protocol and Good Clinical Practice), followed by an online multiplechoice assessment that required a pass mark of $100 \%$. On successful completion of the training package, paramedics received a monetary incentive and a certificate that authorised them to carry the trial drugs and enrol patients. In total, 891 paramedics were offered the chance to participate in the trial, and 521 (59\%) took this opportunity.

As limited evidence exists about the attitudes of emergency medical services clinicians towards their involvement in prehospital research, both within the $\mathrm{UK}^{9}$ and worldwide, ${ }^{1011}$ we sought to gather the views and experiences of the paramedics in our ambulance service (London) who were involved in the trial. Our aim was to obtain their opinions on various aspects of participation in this study including the potential influence of the surrounding controversy and to identify any barriers to recruitment that they experienced.

\section{METHODS}

\section{Study design}

A qualitative study in the form of a questionnaire was designed to explore the attitudes and experiences of London paramedics who were involved in the PARAMEDIC-2 adrenaline trial.

\section{Setting}

The LAS is the main provider of prehospital emergency care across Greater London, UK, spanning approximately 620 square miles and serving an estimated population of 8.8 million people. ${ }^{12}$ Annually, LAS clinicians attend to approximately 1 million incidents, of which over 10000 are OHCAs.

\section{Questionnaire}

The questionnaire consisted of a mixture of 13 closedended and 8 open-ended questions, using a combination of Likert scales and free text fields, to enable collection of both qualitative and quantitative data. Of the 13 closed questions, seven gave the option to provide reasons for their answers in free text. Questions were developed using a combination of RF and JL's research and clinical expertise, plus their experience of managing the PARAMEDIC-2 trial at the LAS. The questionnaire was piloted on a convenience sample of four LAS clinicians to ensure it was readily understandable and produced valid responses. The full questionnaire is available as a supplementary file (online supplementary file 1 ).

Questionnaire completion was voluntary and anonymous. The questionnaire was distributed by a number of methods in an effort to increase the response rate: paper copies were sent through the internal mail system, an electronic copy was distributed via email and a link to the questionnaire online was circulated on social media. The 
survey was open for a 4-week period during November and December 2017.

The study protocol and questionnaire were reviewed and approved under the LAS's R\&D Approval Process. NHS ethical approval was not required in line with the guidance provided by the UK's Health Research Authority. ${ }^{13}$ This study was sponsored by the LAS and was undertaken independently from the PARAMEDIC-2 investigator group.

\section{Respondents}

Questionnaires were sent to all 521 LAS trial-trained paramedics, regardless of whether they had recruited a patient into the study. We did not want to exclude paramedics who may not have had the opportunity to recruit a patient, as we felt that their attitudes towards the trial and research were equally as important as those who had recruited a patient.

\section{Patient and public involvement}

There was no patient and public involvement in this study.

\section{Analysis}

We conducted a complete analysis of free text responses using a grounded theory approach. ${ }^{14} \mathrm{JL}$ initially reviewed and categorised the free text responses, with no prior assumptions. The resulting categories were further reviewed and verified by RI and RF with any changes being agreed by all authors. These categories were then used to describe the responses to the free-text questions.

\section{RESULTS}

\section{Study population}

One hundred and fifty paramedics completed the questionnaire, giving a response rate of $29 \%$. Forty-two responses were returned via paper copy, and 108 were submitted online. Respondents had been qualified as a paramedic for an average of 7 years (range 1-28 years), and $42(28 \%)$ identified themselves as holding a position clinically senior to that of a paramedic (eg, senior paramedic, clinical team leader and advanced paramedic practitioner).

94.7\% of respondents enrolled one or more patients into the PARAMEDIC-2 trial. Four per cent did not directly enrol a patient but were actively involved in one or more resuscitations where the patient was enrolled by another paramedic. Only two respondents did not enrol any patient into the trial.

\section{Findings from the questionnaire}

A summary of responses given to the closed questions are provided in table 1 . Using the responses to both the closed and open ended questions, we derived seven themes that describe the paramedics' attitudes and experiences of participating in the PARAMEDIC-2 trial. These are described in detail below.
Table 1 Responses to the non-free-text questions in the survey $(n=150)$

\begin{tabular}{|c|c|}
\hline Question with response categories & N (\%) \\
\hline \multicolumn{2}{|c|}{$\begin{array}{l}\text { 'How important do you think it is for ambulance services to conduct } \\
\text { research?' }\end{array}$} \\
\hline Very important & $147(98.0)$ \\
\hline Slightly important & $2(1.3)$ \\
\hline Not important at all & $1(0.7)$ \\
\hline \multicolumn{2}{|c|}{$\begin{array}{l}\text { 'How likely would you be to get involved in other prehospital } \\
\text { research in the future?' }\end{array}$} \\
\hline Very likely & $140(93.3)$ \\
\hline A little likely & $9(6.0)$ \\
\hline Not at all likely & $1(0.7)$ \\
\hline \multicolumn{2}{|c|}{$\begin{array}{l}\text { 'Overall would you say your experience of being involved in this trial } \\
\text { was positive or negative?' }\end{array}$} \\
\hline Positive & $146(97.3)$ \\
\hline Negative & $2(1.3)$ \\
\hline Don't know & $2(1.3)$ \\
\hline \multicolumn{2}{|c|}{$\begin{array}{l}\text { 'Were you aware of the wider publicity/controversy surrounding the } \\
\text { PARAMEDIC-2 adrenaline trial?' }\end{array}$} \\
\hline Yes & $86(57.3)$ \\
\hline No & $64(42.7)$ \\
\hline
\end{tabular}

'How did you feel about enrolling patients into the trial without their consent?'

\begin{tabular}{lr} 
Comfortable & $105(70.0)$ \\
\hline Uncomfortable & $8(5.3)$ \\
\hline Indifferent & $36(24.0)$ \\
\hline Did not answer & $1(0.7)$ \\
\hline $\begin{array}{l}\text { 'During recruitment of trial patients, at any point did you explain } \\
\text { their enrolment to relatives or friends on scene?' }\end{array}$ & $24(16.0)$ \\
\hline Yes & $122(81.3)$ \\
\hline No & $4(2.7)$ \\
\hline Did not answer & $148(98.7)$ \\
\hline 'Did you think the methods of PARAMEDIC-2 training (DVD and \\
multiple-choice assessment) was effective?' & $2(1.3)$ \\
\hline Yes & $18(12.0)$ \\
\hline No & $132(88.0)$ \\
\hline 'Is there anything you would have changed about the trial's \\
training?' \\
\hline \begin{tabular}{l} 
Yes \\
\hline No
\end{tabular} \\
\hline 'Would you have completed the trial training if an incentive payment \\
had not been offered?' \\
\hline Yes & $129(86.0)$ \\
\hline No & $14(9.3)$ \\
\hline Did not answer & $7.7)$ \\
\hline
\end{tabular}

Paramedic attitudes towards research

Two questions (Q9 and Q10) explored attitudes towards research. As shown in table 1, 99.3\% of respondents had a positive attitude towards research, reporting that it is either 'very important' (98\%) for ambulance services 
to conduct research or 'slightly important' (1.3\%). One paramedic $(0.7 \%)$ said it is 'not important at all'.

When asked how likely it was that they would get involved in other prehospital research in the future, 99.3\% responded positively (93.3\% reporting 'very likely' and $6 \%$ 'a little likely'). Only one respondent $(0.7 \%)$ replied it is 'not at all likely'.

\section{Experiences of the trial}

This positive attitude was mirrored in paramedics' experiences of being involved in the PARAMEDIC-2 trial, with 97.3\% reporting it as an overall positive experience. Only two $(1.3 \%)$ reported their overall experience to be negative, and a further two answered that they did not know.

\section{Paramedic attitudes towards controversy}

Just over half of respondents were aware of the media publicity/controversy surrounding the PARAMEDIC-2 trial, with the rest being unaware. Of those who were aware, only one reported that it made them less willing, but they still participated and recruited patients nonetheless. The majority of the remaining respondents reported that the publicity/controversy made no difference to their willingness to participate in the trial, and $23.3 \%$ said it made them more willing to participate.

Of those who stated that the controversial publicity either made no difference, or increased their willingness to participate, 33 provided reasons for their answer: $63.6 \%$ cited the importance of the research as the main reason, and $21.2 \%$ reported being motivated by what they considered to be inaccurate negative opinions/publicity towards the trial. One paramedic stated that:

The 'controversy' only reinforced how ill-informed the media is and how increasingly changes in medicine are becoming driven by emotion rather than evidence.

Another paramedic responded that they were:

Aware of some articles in newspapers however these weren't written by medical professionals so I didn't really take any notice.

Another paramedic stated:

With the research that had been done into the possible negative effects of adrenaline in cardiac arrest, it felt so important to me as a paramedic to be part of this trial.

The one respondent who stated that the controversy made them less willing to participate in the trial did not provide a reason for this.

\section{Enrolling without consent}

The majority of paramedics felt comfortable enrolling patients into the trial without seeking prior consent to do so, a quarter reported neutral feelings and 5.3\% felt uncomfortable. Of those who reported being either comfortable or indifferent, 65 provided reasons for their answer. The most commonly cited reason was their perceived importance and necessity of the trial (30.8\%). One paramedic explained:

Understanding the reason the trial is being undertaken reassured me in my decision to become trained and enrol patients.

Other commonly cited reasons included understanding the rationale for not gaining consent in this particular trial $(20 \%)$ and knowledge that the trial had ethical approval (16.9\%). One paramedic answered:

Given all the information in the training covering ethics and consent, I had no concerns about enrolling them.

Others reported feeling comfortable because they knew that patients would be given all proven cardiac arrest treatments (cardiopulmonary resuscitation and defibrillation) where indicated $(9.2 \%)$ and because existing evidence suggests that adrenaline use in cardiac arrest may be harmful $(9.2 \%)$. One paramedic felt neutral because they knew patients had the prior opportunity to opt out of the study.

Of the eight respondents who had felt uncomfortable enrolling patients without consent, five provided reasons. Two felt uncomfortable with specific individual patients and their particular clinical presentation to the ambulance service, rather than feeling generally uncomfortable with the premise of enrolling without consent. Both of these respondents were advanced paramedic practitioners (APPs), the most clinically senior of all respondents. One APP said that they felt uncomfortable enrolling without consent:

Only in the cases... where I felt they would have benefited from inotropic benefit of adrenaline.

One respondent cited the ethics of the trial, referring to it as a 'moral/ethical dilemma'. Another said they felt that the relatives of patients in cardiac arrest would not agree to them being enrolled into a trial, and a further paramedic said they felt uncomfortable because they had always been taught that administering adrenaline was beneficial. Interestingly, the vast majority of those who felt uncomfortable recruiting without consent (6/8) enrolled one or more patients into the trial themselves. The two respondents who felt uncomfortable and did not recruit any patients did not provide reasons for their discomfort.

\section{Explaining the trial to relatives}

Although there was no requirement to explain the trial to relatives or friends when on-scene, 24 paramedics did so on one or more occasion. Most reported occasions where they had actively made the choice to explain the trial to a relative or friend. Another commonly cited reason involved situations where paramedics felt they had no choice due to relatives enquiring about the trial or overhearing clinicians speaking to each other about the trial. 
Barriers to recruitment

The majority of respondents $(60.7 \%)$ did not identify any barriers to recruitment. Fifty-nine $(39.3 \%)$ reported at least one barrier, with other paramedics' attitudes being cited nearly half of the time. One paramedic stated that they:

Several times came up against paramedics who did not agree with the trial, and did not want the patients enrolled.

Another paramedic reported that they were met:

Initially with reluctance on station by other staff, which needed to be overcome often [during an active resuscitation], but to the best of my knowledge, I did not miss an opportunity to enrol a patient as a result, even if I did have to be a little more assertive with colleagues than I might choose to in order to do so.

Three respondents reported negative reactions or attitudes from hospital staff.

Other commonly cited barriers were the need to focus on other tasks $(10.2 \%)$, leaving the trial drugs pack on a vehicle by mistake $(10.2 \%)$, patient ineligibility due to adrenaline having already been administered by other paramedics $(10.2 \%)$ and relatives on scene affecting the paramedic's decision to enrol $(8.2 \%)$. Four paramedics $(8.2 \%)$ reported a lack of opportunity as they did not attend any cardiac arrests during the study period.

\section{Training}

Three questions (Q5, Q6 and Q7) examined attitudes towards the trial training. $98.7 \%$ of respondents thought the training method adopted (35 min DVD presentation and multiple-choice assessment) was effective. Ninetyfive paramedics provided reasons why, the most common being that it was convenient and/or easy to use and understand $(81.1 \%)$. One paramedic stated that it was:

Simple and easy to understand, quick to implement.

Another expressed:

Yes, quite effective. Getting pocket cards helped cement the process and could be carried to help refresh.

Twelve per cent of respondents stated there was something they would have changed, with one paramedic reporting that they would make the participation in the trial 'mandatory for all paramedics'. Just over one-fifth $(22.2 \%)$ reported that they would have preferred the presentation to be shorter. Only two respondents would have preferred face-to-face training. The vast majority of respondents $(90.2 \%)$ stated they would have completed the training regardless of receiving an incentive payment, with one paramedic expressing:

'I believe in doing the best for patients and being involved in this trial clearly would improve cardiac arrest care'.

\section{DISCUSSION}

Paramedics in our study strongly viewed themselves and ambulance services as having an important role to play in clinical research. Our finding that nearly all respondents considered research to be important is consistent with figures reported previously in paramedic surveys in the UK $(92 \%)^{9}$ and the USA (93\% and 98\%). ${ }^{10} 11$ The positively reported experience of being involved in the PARAMEDIC-2 trial, coupled with nearly all respondents claiming they will likely become involved in research in the future, shows a clear appetite for, and understanding of the importance of, prehospital research.

We were particularly interested in how the controversy and negative publicity surrounding the PARAMEDIC-2 trial might have affected paramedics' attitudes towards research. That the controversy made no difference to most paramedics' opinions of the trial, or even motivated them to participate, is interesting. The reasons provided, which largely related to understanding the importance of the research, and a belief that the negative opinions expressed in the media were inaccurate, indicates an understanding and confidence among our sample of paramedics in their contribution to furthering research and improving future patient care. This is further supported by the single paramedic who reported that the controversy made them feel less willing to participate in the trial but who engaged and enrolled patients nonetheless. This attitude reflects similar views among paramedics involved in other clinical trials ${ }^{10}{ }^{11}$ who were also largely driven by the importance of the research and its impact on the society as a whole.

One particularly controversial aspect of the trial surrounded the enrolment of patients into the study without either their prior consent or the proxy consent of a personal representative. However, the majority of paramedics $(70 \%)$ in our study expressed that they were comfortable enrolling patients without consent, mostly because they believed the trial to be necessary and because it had independent ethical approval. This is higher than that reported in a previous study in Yorkshire asking paramedics if it is acceptable in some circumstances to randomise patients before consent, where $51 \%$ were agreeable. ${ }^{9}$ It is important to note that the Yorkshire study did not specifically survey those who had experience of recruiting into trials without gaining consent but was seeking their opinions on doing so, which is a very different situation to ours. Of interest is the fact that, in our study, three-quarters of those who felt uncomfortable enrolling patients without consent continued to do so. This goes some way to demonstrating the complexity of the question regarding a paramedic's level of comfort enrolling patients without consent and their investment in delivering research.

The fact that the majority of respondents felt comfortable enrolling patients without consent could partly be explained by the training package that we provided to them. Schmidt et $a l^{10}$ suggest that education for ambulance staff on the legal guidelines around enrolling 
patients into clinical trials without their consent could be beneficial. In the LAS, the trial's training package covered consent in much detail; we explained why consent was unfeasible to obtain for these patients and that the trial had been reviewed and approved by an independent ethics committee.

However, of course, the overall comfort that the majority of our respondents had with enrolling patients without consent could also be explained by the fact that participation in the trial was voluntary, and thus, they were self-selected. Arguably only paramedics who were comfortable with the concept would have completed the trial's training, which qualified them to participate. This self-selection bias is an important limitation of our study.

Due to the urgent nature with which treatment had to be given, it was not practical to obtain informed consent from the patient or legal representative. Approval was provided by the Research Ethics Committee to enrol patients and seek deferred consent when the initial emergency had passed. Although there was no obligation on the part of the enrolling paramedic to explain the trial to relatives on scene, a small proportion recalled situations where they did explain the trial to relatives or friends. Sometimes this was because they actively chose to, and at other times they felt obliged to either because they were directly asked or became aware that relatives had overheard discussions between clinicians about the trial.

We also wanted to explore any barriers to patient recruitment that paramedics experienced. Almost half of the respondents who reported having experienced barriers to recruitment identified other (nontrial trained) paramedics' attitudes as the main cause. This response provides a small level of insight into the contrasting views and opinions of some paramedics who were not involved in the trial. Of course, non-trial trained paramedics would not have been party to all the background literature and ethical considerations that were provided through our training package. A survey of paramedics who did not volunteer to participate in the trial may have provided useful information about why and how to engage this cohort in the future. This is particularly important for research into cardiac arrest where resuscitation efforts usually require multiple clinicians, who will likely have differing beliefs and opinions, all working together. As these differences could present barriers and challenges to patient enrolment, developing strategies for connecting with a non-engaged cohort is an area for future investigation.

Our questionnaire return rate of $29 \%$, which is in line with previously reported response rates, ${ }^{9}{ }^{15} 16$ provides a level of confidence in our findings. However, we must nonetheless interpret our findings with some caution as they are specific to a sample of paramedics who were trial trained within the LAS. Other ambulance services participated in PARAMEDIC-2, and while we have no reason to expect their experiences to differ greatly, our findings are limited to only one geographical area of the trial. Moreover, our survey did not collect detailed demographic information from the study participants, which could have rendered it more insightful.

\section{CONCLUSION}

Our findings demonstrate a healthy appetite for involvement in prehospital research among LAS paramedics, and supports similar research conducted elsewhere. Furthermore it demonstrates that this enthusiasm and willingness to participate continues to exist even in a situation where consent to recruit is not sought from either the patient or a relative. This is an important finding demonstrating that such research can be undertaken successfully by paramedics in the prehospital environment.

Acknowledgements We would like to thank all paramedics who completed and returned a questionnaire.

Contributors RF conceived and designed the study. RF and $\mathrm{JL}$ designed the questionnaire and wrote the manuscript. JL collected the data from the survey. JL and RI undertook analyses. RI contributed to revisions to the manuscript.

Funding The PARAMEDIC-2 trial was funded by the Health Technology Assessment Programme of the National Institute for Health Research (12/127/126).

Disclaimer The funders had no role in the trial design, data collection or analysis, or in the writing of this report.

Competing interests None declared.

Patient consent for publication Not required.

Ethics approval Approval was provided by the Research Ethics Committee to enrol patients and seek deferred consent when the initial emergency had passed.

Provenance and peer review Not commissioned; externally peer reviewed.

Data availability statement № data are available.

Open access This is an open access article distributed in accordance with the Creative Commons Attribution Non Commercial (CC BY-NC 4.0) license, which permits others to distribute, remix, adapt, build upon this work non-commercially, and license their derivative works on different terms, provided the original work is properly cited, appropriate credit is given, any changes made indicated, and the use is non-commercial. See: http://creativecommons.org/licenses/by-nc/4.0/.

ORCID iD

Rachael T Fothergill http://orcid.org/0000-0003-1341-6200

\section{REFERENCES}

1 Perkins GD, Ji C, Deakin CD, et al. A randomized trial of epinephrine in out-of-hospital cardiac arrest. N Engl J Med 2018;379:711-21.

2 Jacobs IG, Finn JC, Jelinek GA, et al. Effect of adrenaline on survival in out-of-hospital cardiac arrest: a randomised double-blind placebocontrolled trial. Resuscitation 2011;82:1138-43.

3 Olasveengen TM, Sunde K, Brunborg C, et al. Intravenous drug administration during out-of-hospital cardiac arrest: a randomized trial. JAMA 2009;302:2222-9.

4 Hagihara A, Hasegawa M, Abe T, et al. Prehospital epinephrine use and survival among patients with out-of-hospital cardiac arrest. JAMA 2012;307:1161-8.

5 Perkins GD, Quinn T, Deakin CD, et al. Pre-Hospital assessment of the role of adrenaline: measuring the effectiveness of drug administration in cardiac arrest (PARAMEDIC-2): trial protocol. Resuscitation 2016;108:75-81.

6 Smith R, Hill G. Heart patients to be given placebo by paramedics in controversial trial, 2014. Available: https://www.telegraph.co.uk/ news/health/news/11028692/Heart-patients-to-be-given-placeboby-paramedics-in-controversial-trial.html [Accessed 11 Jul 2018].

7 Mundasad S. Resuscitation drug adrenaline questioned in cardiac arrest, 2014. Available: https://www.bbc.co.uk/news/health28770885 [Accessed 11 Jul 2018].

8 Borland S. Paramedics to give dummy drug for heart attacks: controversial trial will see patients given placebo instead of adrenaline when their heart has stopped, 2014. Available: http:// 
www.dailymail.co.uk/news/article-2723408/Paramedics-dummydrug-heart-attacks-Controversial-trial-patients-given-placeboinstead-adrenaline-heart-stopped.html [Accessed 11 Jul 2018].

9 Hargreaves K, Goodacre S, Mortimer P. Paramedic perceptions of the feasibility and practicalities of prehospital clinical trials: a questionnaire survey. Emerg Med $J$ 2014;31:499-504.

10 Schmidt TA, Nelson M, Daya M, et al. Emergency medical service providers' attitudes and experiences regarding enrolling patients in clinical research trials. Prehosp Emerg Care 2009;13:160-8.

11 Newington L, Metcalfe A. Factors influencing recruitment to research: qualitative study of the experiences and perceptions of research teams. BMC Med Res Methodol 2014;14:10.
12 London Ambulance Service NHS Trust. Cardiac arrest annual report 2017-2018, 2018. Available: https://www.londonambulance.nhs.uk/ about-us/our-publications/ [Accessed Mar 2019].

13 NHS health research authority. Available: http://www.hradecisiontools.org.uk/ethics/ [Accessed Mar 2019].

14 Glaser BG, Strauss AL. The Discovery of Grounded Theory:Strategies for Qualitative Research. Chicago: Aldine Publishing, 1967.

15 Jasti J, Fernandez AR, Schmidt TA, et al. Ems provider attitudes and perceptions of Enrolling patients without consent in prehospital emergency research. Prehosp Emerg Care 2016;20:22-7.

16 Heick JD, Farris JW. Survey of methods used to determine if a patient has a deep vein thrombosis: an exploratory research report. Physiother Theory Pract 2017;33:733-42. 